\title{
HIV Dynamics Under Antiretroviral Treatment with Interactivity
}

\author{
Beatriz Laiate $^{1(\otimes)}\left(\mathbb{D}\right.$, Francielle Santo Pedro ${ }^{2}(\mathbb{D})$, Estevão Esmi $^{1(\otimes)}(\mathbb{D}$, \\ and Laécio Carvalho de $\operatorname{Barros}^{1(\otimes)}(\mathbb{D}$ \\ 1 Department of Applied Mathematics, University of Campinas, \\ Campinas, SP 13083-970, Brazil \\ beatrizlaiate@gmail.com, eelaureano@gmail.com, laeciocb@ime.unicamp.br \\ 2 Multidisciplinary Department, Federal University of São Paulo, \\ Osasco, SP 06110-295, Brazil \\ fsimoes@unifesp.br
}

\begin{abstract}
This manuscript presents a model for HIV dynamics of seropositive individuals under antiretroviral treatment described from fuzzy set theory by two different approaches considering interactivity: differential equation with interactive derivative and differential equation with Fréchet derivative. It also establishes an identity between interactive derivative and fuzzy Fréchet derivative. With this identity, we establish when the solutions of the two differential equations coincide. Lastly, we present biological interpretations for both cases.
\end{abstract}

Keywords: HIV · Antiretroviral treatment $\cdot$ Fuzzy interactive differential equation $\cdot$ Fréchet derivative $\cdot$ Fuzzy interactive derivative

\section{Introduction}

HIV dynamics considering antiretroviral treatment (ART) has already been studied in several articles $[11,16,18,20]$. The major target of HIV are CD4+ $\mathrm{T}$ cells, a class of immune cells. Antiviral drugs act blocking biological processes involved in life cycle virus into cell cytoplasm. Most common therapies combine protease inhibitors and reverse transcriptase inhibitors. The first ones block HIV protease, so that noninfectious viral particles start being produced by infected T-cells, and the last ones prevent the successfully infection of T-cells. Data obtained in previous studies $[17,18]$ show that under combination of protease inhibitors and RT inhibitors, a viral decline in the bloodstream is followed by the increase of CD4+ population are expected.

Fuzzy set theory applied to HIV dynamics under treatment was already studied using fuzzy rule-based systems [12-14] and Choquet Calculus [15], both considering an intracellular delay assigned maily to the pharmacological delay, defined as the interval of time required for the absorption of the antiviral drugs in the bloodstream. Viral dynamics represented as an interactive process is a new 
approach in the literature and provides results subject to new interpretations for already known HIV models.

The existence of memory in biological processes was already considered in previous studies $[1,2]$. Current studies consider the existence of memory in biological processes, in particular, in the dynamics of HIV, when representing them by fuzzy fractional derivatives with interactivity [25]. Autocorrelated processes take into account the dependence between their states in consecutive instants. This type of approach allows us to describe processes that are hidden or inherent to the phenomenon when considering a memory coefficient $f_{t, h}$. This coefficient changes with the process and is determined by the current moment. Thus, there may exist memories with different properties for different periods of time. Therefore, it is necessary a derivative operator that incorporates the memory of the system, being responsible for its variation.

There are various different theories of fuzzy differential equations for fuzzyset-valued functions $[4,5,21,24]$, that is, functions $f:[a, b] \rightarrow \mathbb{R}_{\mathcal{F}}$, where $\mathbb{R}_{\mathcal{F}}$ is the space of fuzzy numbers, that is, fuzzy subsets of $\mathbb{R}$ whose $\alpha$-levels are closed intervals in real line [3]. In this work we will represent an HIV intracellular model throught two interactive derivatives defined in an autocorrelated process. In particular, we assume that it is a linearly correlated process [8,23].

Firstly we describe the dynamics via the linearly correlated derivative, based on the difference between fuzzy sets, that is, the difference obtained from possibility distributions of fuzzy sets envolved [26]. This derivative provides two possible behaviors for the solution, expansive or contractive. In the first case, the fuzziness of the solution increase with time while in the second one, it decreases. In this work, the fuzziness is measured accordingly to the diameter of the fuzzy number. The larger the diameter of the fuzzy number, the greater its fuzziness.

On the other hand, fuzzy differential equation via Fréchet derivative is based on the isomorphim $\Psi_{A}: \mathbb{R}^{2} \rightarrow \mathbb{R}_{\mathcal{F}(A)}$, where $\mathbb{R}_{\mathcal{F}(A)}$ is the set of all fuzzy numbers linearly correlated to $A \in \mathbb{R}_{\mathcal{F}}[10]$. This allows us to define the induced sum and scalar multiplication in $\mathbb{R}_{\mathcal{F}(A)}$ given by $B+{ }_{A} C=\Psi_{A}\left(\Psi_{A}{ }^{-1}(B)+\Psi_{A}{ }^{-1}(C)\right)$ and $\eta \cdot{ }_{A} B=\Psi_{A}\left(\eta \Psi_{A}{ }^{-1}(B)\right), \forall B, C \in \mathbb{R}_{\mathcal{F}(A)}$ and $\eta \in \mathbb{R}$. With this operations it is possible to confer a Banach space structure to the space $\mathbb{R}_{\mathcal{F}(A)}$ and, therefore, develop a calculus theory for the family of fuzzy functions linearly correlated to $A \in \mathbb{R}_{\mathcal{F}}$, as it was done in $[22]$.

This work is structured as follows. Section 2 provides the mathematical concepts necessary to understand the development of this work. Section 3 presents HIV dynamics in two different approaches: via L-derivative and Fréchet derivative. Section 4 presents final comments.

\section{Mathematical Background}

A fuzzy subset $A$ of $\mathbb{R}$ is described by its membership function $\mu_{A}: \mathbb{R} \rightarrow[0,1]$, where $\mu_{A}(x)$ is the degree of membership of $x$ in $A$. The $\alpha$-cuts of $A$ are subsets of $\mathbb{R}$ given by $[A]_{\alpha}=\{x \in \mathbb{R}: A(x) \geq \alpha\}$, for $\alpha \in(0,1]$, and $[A]_{0}$ is the closure of the support of $A$, that is, $[A]_{0}=\overline{\{x \in \mathbb{R}: A(x)>0\}}$. 
The fuzzy subset $A$ of $\mathbb{R}$ is a fuzzy number if all the $\alpha$-cuts are closed and nonempty intervals of $\mathbb{R}$ and the support of $A$ is bounded [3]. The set of all fuzzy numbers is denoted by $\mathbb{R}_{\mathcal{F}}$. We define the $\operatorname{diam}(A)=\left|a_{0}^{+}-a_{0}^{-}\right|$, where $a_{0}^{-}$and $a_{0}^{+}$are the endpoints of $[A]_{0}$, for all $A \in \mathbb{R}_{\mathcal{F}}$.

Let $a, b, c \in \mathbb{R}$ such that $a \leq b \leq c$, a triangular fuzzy number $A$ is a wellknown example of fuzzy number given by the following membership function:

$$
\mu_{A}(x)=\left\{\begin{array}{cl}
0, & x \leq a \text { or } x \geq b \\
\frac{x-a}{b-a} \wedge \frac{c-x}{c-b} \text { otherwise, }
\end{array}\right.
$$

where $\wedge$ is the minimum operator. In this case, we denote $A$ by the symbol $(a ; b ; c)$.

Next, we recall some concepts necessary to understand the theory of interactive derivative in the space of fuzzy numbers.

A possibility distribution on $\mathbb{R}^{n}$ is a fuzzy subset $J$ of $\mathbb{R}^{n}$ with membership function $\mu_{J}: \mathbb{R}^{n} \rightarrow[0,1]$ satisfying $\mu_{J}\left(x_{0}\right)=1$ for some $x_{0} \in \mathbb{R}^{n}$. The family of possibility distributions of $\mathbb{R}^{n}$ will be denoted by $\mathcal{F}_{J}\left(\mathbb{R}^{n}\right)$.

Definition 1 [6]. Let $A, B \in \mathbb{R}_{\mathcal{F}}$ and $J \in \mathcal{F}_{J}\left(\mathbb{R}^{2}\right)$. Then $\mu_{J}$ is a joint possibility distribution of $A$ and $B$ if $\max _{y} \mu_{J}(x, y)=\mu_{A}(x)$ and $\max _{x} \mu_{J}(x, y)=\mu_{B}(y)$, for any $x, y \in \mathbb{R}$.

In this case, $\mu_{A}$ and $\mu_{B}$ are called marginal possibility distributions of $J$.

Definition 2 [9]. The fuzzy numbers $A$ and $B$ are said to be non-interactive if and only if their joint possibility distribution $J$ satisfies the relationship $\mu_{J}(x, y)=\min \left(\mu_{A}(x), \mu_{B}(y)\right)$ for all $x, y \in \mathbb{R}$. Otherwise, are said to be interactive.

Definition $3[6,9]$. The fuzzy numbers $A$ and $B$ are said to be completely correlated if there exist $q, r \in \mathbb{R}, q \neq 0$ such that joint possibility distribution is defined by

$$
\mu_{C}(x, y)=\mu_{A}(x) \chi_{q x+r=y}(x, y)=\mu_{B}(y) \chi_{q x+r=y}(x, y)
$$

where $\chi_{q x+r}(x, y)$ represents the characteristic function of the line $\left\{(x, y) \in \mathbb{R}^{2}\right.$ : $q x+r=y\}$.

Definition 4 [8]. Two fuzzy numbers $A$ and $B$ are said linearly correlated if there exist $q, r \in \mathbb{R}$ such that their $\alpha$-levels satisfy $[B]_{\alpha}=q[A]_{\alpha}+r$ for all $\alpha \in[0,1]$. In this case, we write $B=q A+r$.

Definition 5. The four arithmetic operations between linearly correlated fuzzy numbers are defined, in levels, by:

- $\left[B+_{L} A\right]_{\alpha}=(q+1)[A]_{\alpha}+r, \forall \alpha \in[0,1]$;

- $\left[B-{ }_{L} A\right]_{\alpha}=(q-1)[A]_{\alpha}+r, \forall \alpha \in[0,1]$;

- $\left[B \cdot{ }_{L} A\right]_{\alpha}=\left\{q x_{1}^{2}+r x_{1} \in \mathbb{R} \mid x_{1} \in[A]_{\alpha}\right\}, \forall \alpha \in[0,1]$;

- $\left[B \div{ }_{L} A\right]_{\alpha}=\left\{q+\frac{r}{x_{1}} \in \mathbb{R} \mid x_{1} \in[A]_{\alpha}\right\}, \forall \alpha \in[0,1]$. 
The Pompeiu-Hausdorff distance $d_{\infty}: \mathbb{R}_{\mathcal{F}}^{n} \times \mathbb{R}_{\mathcal{F}}^{n} \rightarrow \mathbb{R}_{+} \cup\{0\}$ is defined by

$$
d_{\infty}(A, B)=\sup _{0 \leq \alpha \leq 1} d_{H}\left([A]_{\alpha},[B]_{\alpha}\right)
$$

where $d_{H}$ is the Pompeiu-Hausdorff distance for sets in $\mathbb{R}^{n}$. If $A$ and $B$ are fuzzy numbers, then (3) becomes

$$
d_{\infty}(A, B)=\sup _{0 \leq \alpha \leq 1} \max \left\{\left|a_{\alpha}^{-}-b^{-} \alpha\right|,\left|a_{\alpha}^{+}-b_{\alpha}^{+}\right|\right\} .
$$

The derivative enunciated in this subsection is related to an autocorrelated process $F:[a, b] \rightarrow \mathbb{R}_{\mathcal{F}}$, that is, for $h$ with absolute value sufficiently small, $F(t+h)=q(h) F(h)+r(h)$, for all $t \in[a, b], q(h), r(h) \in \mathbb{R}$. This formula means that $[F(t+h)]_{\alpha}=q(h)[F(t)]_{\alpha}+r(h), \forall \alpha \in[0,1]$.

Definition 6 [8]. Let $F:[a, b] \rightarrow \mathbb{R}_{\mathcal{F}}$ be a fuzzy-number-valued function and for each $h$ with absolute value sufficiently small, let $F\left(t_{0}+h\right)$ and $F\left(t_{0}\right)$ with $t_{0} \in[a, b]$ be linearly correlated fuzzy numbers. $F$ is called L-differentiable at $t_{0}$ if there exists a fuzzy number $D_{L} F\left(t_{0}\right) \in \mathbb{R}_{\mathcal{F}}$ such that the limit

$$
\lim _{h \rightarrow 0} \frac{F\left(t_{0}+h\right)-{ }_{L} F\left(t_{0}\right)}{h}
$$

exists and is equal to $D_{L} F\left(t_{0}\right)$, using the metric $d_{\infty} . D_{L} F\left(t_{0}\right)$ is called linearly correlated fuzzy derivative of $F$ at $t_{0}$. At the endpoints of $[a, b]$ we consider only one-sided derivative.

The next theorem provides a practical formula to calculate the L-derivative of an autocorrelated process.

Theorem 1 [8]. Let $F:[a, b] \rightarrow \mathbb{R}_{\mathcal{F}}$ be L-differentiable in $t_{0}$ and $F_{\alpha}\left(t_{0}\right)=$ $\left[F\left(t_{0}\right)\right]_{\alpha}=\left[f_{\alpha}^{-}\left(t_{0}\right), f_{\alpha}^{+}\left(t_{0}\right)\right]$, for all $\alpha \in[0,1]$. Then $f_{\alpha}^{-}$and $f_{\alpha}^{+}$are differentiable in $t_{0}$ and for all $|h|<\delta$, for some $\delta>0$ and

$$
\left[D_{L} F\left(t_{0}\right)\right]_{\alpha}=\left\{\begin{array}{cc}
i . & {\left[\left(f_{\alpha}^{-}\right)^{\prime}\left(t_{0}\right),\left(f_{\alpha}^{+}\right)^{\prime}\left(t_{0}\right)\right] \quad \text { if } q(h) \geq 1} \\
\text { ii. }\left[\left(f_{\alpha}^{+}\right)^{\prime}\left(t_{0}\right),\left(f_{\alpha}^{-}\right)^{\prime} t_{0}\right] & \text { if } 0<q(h) \leq 1 \\
\text { iii. }\left[\left(f_{\alpha}^{-}\right)^{\prime}\left(t_{0}\right),\left(f_{\alpha}^{-}\right)^{\prime}\left(t_{0}\right)\right] & \text { if } q(h)<0
\end{array}\right.
$$

where $D_{L} F\left(t_{0}\right)$ is the L-derivative.

Next, we present important results related to the theory of calculus developed in the space of fuzzy numbers linearly correlated to a given fuzzy number $A$.

A fuzzy number $A \in \mathbb{R}_{\mathcal{F}}$ is said to be symmetric with respect to $x \in \mathbb{R}$ if $A(x-y)=A(x+y), \forall y \in \mathbb{R}$, and it is said to be non-symmetric if there exists no $x$ such that $A$ is symmetric. For example, the fuzzy number $A=(-1 ; 0 ; 1)$ is symmetric with respect to 0 and the fuzzy number $B=(2 ; 3 ; 5)$ is not symmetric.

Given $A \in \mathbb{R}_{\mathcal{F}}$, we can define the operator $\Psi_{A}: \mathbb{R}^{2} \rightarrow \mathbb{R}_{\mathcal{F}}$ so that $\Psi_{A}(q, r)=$ $q A+r$, that is, the image of the pair $(q, r)$ is the fuzzy number $\Psi_{A}(q, r)$ whose 
$\alpha$-cuts are given by $\left[\Psi_{A}(q, r)\right]_{\alpha}=\left\{q x+r \in \mathbb{R} \mid x \in[A]_{\alpha}\right\}=q[A]_{\alpha}+r$. The range of the operator $\Psi_{A}$ is denoted by $\mathbb{R}_{\mathcal{F}(A)}=\left\{\Psi_{A}(q, r) \mid(q, r) \in \mathbb{R}^{2}\right\}$. This operator defines an isomorphism between $\mathbb{R}^{2}$ and $\mathbb{R}_{\mathcal{F}(A)}$ whenever $A$ is a non-symmetric fuzzy number [10]. Since $\mathbb{R}^{2}$ is a Banach space, we can conclude that $\mathbb{R}_{\mathcal{F}(A)}$ is also a Banach space.

Let $A$ be a non-symmetric fuzzy number. We say that a fuzzy-number-valued function $f:[a, b] \rightarrow \mathbb{R}_{\mathcal{F}(A)} \subseteq \mathbb{R}_{\mathcal{F}}$ is continuous in $\mathbb{R}_{\mathcal{F}(A)}$ when it is continuous with respect to the norm $\|\cdot\|_{\Psi_{A}}$. These functions are called $A$-linearly correlated fuzzy processes. The following lemma characterizes this type of function.

Lemma 1 [10]. Let $A \in \mathbb{R}_{\mathcal{F}}$ be non-symmetric. There exists unique $(q, r)=p$ : $\mathbb{R} \rightarrow \mathbb{R}^{2}$ such that $f=\Psi_{A} \circ p$.

Theorem 2 [10]. Let $B=\Psi_{A}(q, r) \in \mathbb{R}_{\mathcal{F}}$ for some $A \in \mathbb{R}_{\mathcal{F}}$ and some pair $(q, r) \in \mathbb{R}^{2}$ with $q \neq 0$. Then the ranges of operators $\Psi_{A}$ and $\Psi_{B}$ are identical, that is, $\mathbb{R}_{\mathcal{F}(A)}=\mathbb{R}_{\mathcal{F}(B)}$.

The next theorem establishes sufficient and necessary conditions to an $A$ linearly correlated fuzzy process to be continuous.

Theorem 3 [22]. Let $A$ be non-symmetric and $f=\Psi_{A} \circ p:[a, b] \longrightarrow \mathbb{R}_{\mathcal{F}(A)}$. The function $f:[a, b] \longrightarrow \mathbb{R}_{\mathcal{F}(A)}$ is continuous if, and only if, $p:[a, b] \rightarrow \mathbb{R}^{2}$ is continuous.

Since, for $A \in \mathbb{R}_{\mathcal{F}}$ non-symmetric, $\mathbb{R}_{\mathcal{F}(A)}$ is a Banach space, it is possible to define the Fréchet derivative of $f$ as it was done in [10]. The next proposition presents a necessary and sufficient condition to $f: \mathbb{R} \rightarrow \mathbb{R}_{\mathcal{F}(A)}$ to be Fréchet differentiable.

Proposition 1 [10]. Let $A \in \mathbb{R}_{\mathcal{F}}$ be non-symmetric and $f:[a, b] \longrightarrow \mathbb{R}_{\mathcal{F}(A)} \subset$ $\mathbb{R}_{\mathcal{F}}$. The function $f$ is Fréchet differentiable at $t$ if, and only if, $\Psi_{A}^{-1} \circ f:[a, b] \longrightarrow$ $\mathbb{R}^{2}$ is Fréchet differentiable at $t$.

Theorem 4 [10]. Let $A \in \mathbb{R}_{\mathcal{F}}$ be non-symmetric, the functions $q, r: \mathbb{R} \rightarrow \mathbb{R}$ and $f: \mathbb{R} \rightarrow \mathbb{R}_{\mathcal{F}(A)}$ such that $f(t)=\Psi_{A}(q(t), r(t)), \forall t \in \mathbb{R}$. The function $f$ is Fréchet differentiable (F-differentiable) at $t \in \mathbb{R}$ if and only if $q^{\prime}(t)$ and $r^{\prime}(t)$ exist. Additionally, the $F$-derivative of $f$ at $t$ is given by $f^{\prime}(t, h)=\Psi_{A}\left(q^{\prime}(t) h, r^{\prime}(t) h\right)$, $\forall h \in \mathbb{R}$.

Fuzzy interactive derivatives studied in this paper can be related algebraically throught Theorem 5 .

Theorem 5. Let $A$ be a non-symmetric fuzzy number, $f: \mathbb{R} \rightarrow \mathbb{R}_{\mathcal{F}(A)}$ given by $f(t)=\Psi_{A}(p(t), q(t))$, where $p, q$ are real functions for all $t \in \mathbb{R}$. Then $f$ is Fréchet differentiable if, and only if, $f$ is L-differentiable, where the L-derivative is the interactive derivative [8]. Moreover, the Fréchet derivative of $f$ coincide with the L-derivative of $f$, that is

$$
\Psi_{A}\left(p^{\prime}(t), q^{\prime}(t)\right)=D_{L} f(t), \forall t \in \mathbb{R} .
$$


Proof. Let $f: \mathbb{R} \rightarrow \mathbb{R}_{\mathcal{F}(A)}$ be given by $f(t)=p(t) A+q(t)$, for all $t \in \mathbb{R}$ such that $f(t) \neq \mathbb{R}, \forall t \in \mathbb{R}$.

For $h \in \mathbb{R}$, we have that $f(t+h)=p(t+h) A+q(t+h)$ and, therefore,

$$
f(t+h)=\left(\frac{p(t+h)}{p(t)}\right) f(t)+\left(q(t+h)-p(t+h) \frac{q(t)}{p(t)}\right) .
$$

Denoting $\tilde{p}(h)=\frac{p(t+h)}{p(t)}$ and $\tilde{q}(h)=q(t+h)-\frac{p(t+h) q(t)}{p(t)}$, we may write

$$
f(t+h)=\tilde{p}(h) f(t)+\tilde{q}(h), \forall h \in \mathbb{R} .
$$

Thus, if $f(t)=\Psi_{A}(p(t), q(t)), \forall t \in \mathbb{R}, f$ represents a linearly correlated fuzzy process. So, we have that

$$
f(t+h){ }_{-} f(t)=(\tilde{p}(h)-1) f(t)+\tilde{q}(h)
$$

for all $t, h \in \mathbb{R}$. If $f$ is Fréchet differentiable, then the derivatives $p^{\prime}, q^{\prime}: \mathbb{R} \rightarrow \mathbb{R}$ exist for all $t \in \mathbb{R}$, according to Theorem 4 . Moreover,

$$
\lim _{h \rightarrow 0} \tilde{p}(h)=\lim _{h \rightarrow 0} \frac{p(t+h)}{p(t)}=1
$$

and

$$
\lim _{h \rightarrow 0} \tilde{q}(h)=\lim _{h \rightarrow 0} q(t+h)-q(t) \frac{p(t+h)}{p(t)}=0,
$$

once $p$ and $q$ are continuous in $t$. Therefore, $\lim _{h \rightarrow 0}\left[f(t+h){ }_{L} f(t)\right]=\lim _{h \rightarrow 0}[(\tilde{p}(h)-$ 1) $f(t)+\tilde{q}(h)]=0$.

For all $\alpha \in[0,1]$ we have that

$$
\lim _{h \rightarrow 0} \frac{\left[f(t+h)-{ }_{L} f(t)\right]_{\alpha}}{h}=\lim _{h \rightarrow 0}\left[\left(\frac{\tilde{p}(h)-1}{h}\right)[f(t)]_{\alpha}+\left(\frac{q(\tilde{h})}{h}\right)\right]
$$

Note that

$$
\lim _{h \rightarrow 0}\left(\frac{\tilde{p}(h)-1}{h}\right)=\lim _{h \rightarrow 0} \frac{\left(\frac{p(t+h)}{p(t)}-1\right)}{h}=\frac{p^{\prime}(t)}{p(t)}
$$

and

$$
\lim _{h \rightarrow 0} \frac{\tilde{q}(h)}{h}=\lim _{h \rightarrow 0} \frac{q(t+h)-\frac{p(t+h) q(t)}{p(t)}}{h}=q^{\prime}(t)-\frac{q(t) p^{\prime}(t)}{p(t)}
$$

for all $t \in \mathbb{R}$. Therefore,

$$
\begin{aligned}
{\left[D_{L} f(t)\right]_{\alpha} } & =\lim _{h \rightarrow 0}\left(\frac{\tilde{p}(h)-1}{h}\right)[f(t)]_{\alpha}+\lim _{h \rightarrow 0}\left(\frac{\tilde{q}(h)}{h}\right) \\
& =\frac{p^{\prime}(t)}{p(t)}[f(t)]_{\alpha}+q^{\prime}(t)-\frac{q(t) p^{\prime}(t)}{p(t)}
\end{aligned}
$$

for all $t \in \mathbb{R}$ and $\alpha \in[0,1]$.

Since $[f(t)]_{\alpha}=p(t)[A]_{\alpha}+q(t)$ for all $\alpha \in[0,1]$, we have

$$
D_{L}(f)(t)=p^{\prime}(t) A+q^{\prime}(t)=\Psi_{A}\left(p^{\prime}(t), q^{\prime}(t)\right), \forall t \in \mathbb{R} .
$$

The converse implication is immediate, from Theorem 1. 


\section{HIV Dynamics Under Antiretroviral Treatment (ART)}

Data obtained in various studies [17] appear to show that the decay of plasma viraemia in bloodstream is approximately exponential after the patient was placed on a potent antiretroviral drug. One of the simplest models of viral dynamics consider the effect of antiretroviral in viral population as in Eq. (19)

$$
\frac{d V}{d t}=P-c V, V(0)=V_{0}
$$

where $P$ is the rate of virus production, $c$ is the clearance rate and $V=V(t)$ is the virus concentration in bloodstream. This model assumes that the treatment is initiated at $t=0$ and that the efficiency of the treatment is partial, so that $P>0$. With this assumption, virus decay is not perfectly "exponential", so that the solution of (19) is given by

$$
V(t)=V_{0} e^{-c t}+\frac{P}{c}-\frac{P}{c} e^{-c t} .
$$

Equation (20) means viral load declines whenever $V_{0}>\frac{1}{c}$, where $\frac{1}{c}$ is the average life time of the viruses when the efficiency of the treatment is total, that is, $P=0$.

Next, we will establish two different fuzzy approaches to HIV dynamics described in (19), both considering interactivity into the process $V$.

\subsection{Fuzzy Interactive Differential Equation via L-Derivative}

Analysis of models considering CD4+ cell population suggests that, when starting the treatment, viral load is related to parameters such as virus and infected cells elimination rates, as well as and the number of viral particles produced by each infected CD4+ cell [19]. The uncertainty of these rates suggests that the viral load may be well represented when $V$ is a fuzzy number.

We will consider that viral dynamics described in (19) is an autocorrelated fuzzy process. According to Definition 6 , this means that for each $h$ with absolute value sufficiently small, $V(t+h)=q(h) V(t)+r(h)$ for all $t \geq 0$, where $q, r$ are real functions. Then, the corresponding Fuzzy Initial Value Problem (FIVP) via L-derivative is given by

$$
\left\{\begin{array}{l}
V_{L}^{\prime}(t)=P-{ }_{L} c V(t) \\
V(0)=V_{0} \in \mathbb{R}_{\mathcal{F}},
\end{array}\right.
$$

where $P, c>0$ are real constants. 
According to Theorem 1, there are two cases to consider:

i) $q \geq 1$ :

In this case, the solution of $(21)$ for $V(t)=\left[v_{\alpha}^{-}(t), v_{\alpha}^{+}(t)\right]$, in levels, is given by

$$
[V(t)]_{\alpha}=\left[k_{1}^{\alpha} e^{c t}+k_{2}^{\alpha} e^{-c t}+\frac{P}{c},-k_{1}^{\alpha} e^{c t}+k_{2}^{\alpha} e^{-c t}+\frac{P}{c}\right],
$$

where $k_{1}^{\alpha}=-\frac{v_{\alpha}^{+}(0)-v_{\alpha}^{-}(0)}{2}$ and $k_{2}^{\alpha}=\frac{v_{\alpha}^{+}(0)+v_{\alpha}^{-}(0)}{2}-\frac{P}{c}$. As expected in the expansive solution, we have that $\lim _{t \rightarrow \infty} \operatorname{diam}\left(V_{0}\right)=\lim _{t \rightarrow \infty}\left|v_{0}^{+}(0)-v_{0}^{-}(0)\right| e^{c t}=$ $+\infty$, that is, the fuzziness raises with time, as depicted in Fig. 1.

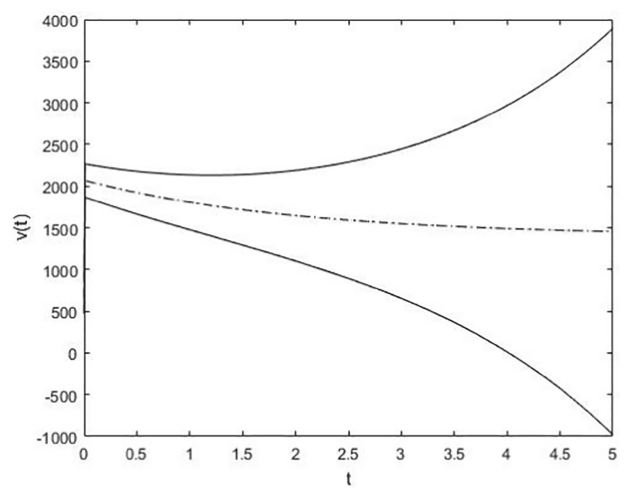

Fig. 1. Viral load as an expansive fuzzy process for $P=700$ virions/day, $c=0.5 /$ day and $V_{0}=(470 ; 670 ; 870)$ virions $[18,19]$. Only positive values for $\mathrm{V}$ should be considered.

ii) $0<q<1$ :

In this case, the solution of $(21)$ for $V(t)_{\alpha}=\left[v_{\alpha}^{-}(t), v_{\alpha}^{+}(t)\right]$, in levels, is given by

$$
[V(t)]_{\alpha}=\left[\left(v_{\alpha}^{-}(0)-\frac{P}{c}\right) e^{-c t}+\frac{P}{c},\left(v_{\alpha}^{+}(0)-\frac{P}{c}\right) e^{-c t}+\frac{P}{c}\right] .
$$

As expected in the contractive solution, $\lim _{t \rightarrow \infty} v_{\alpha}^{-}(t)=\lim _{t \rightarrow \infty} v_{\alpha}^{+}(t)=\frac{P}{c}$ and, therefore, $\operatorname{diam}(V(t)) \rightarrow 0$, that is, the fuzziness vanishes with time. We have that $v_{\alpha}^{-}(t), v_{\alpha}^{+}(t) \geq 0, \forall t \geq 0$ if, and only if, $v_{\alpha}^{-}(0), v_{\alpha}^{+}(0) \geq \frac{P}{c}\left(1-e^{c t}\right)$ for all $t \geq 0$, that is, $v_{\alpha}^{-}(0), v_{\alpha}^{+}(0) \geq \frac{P}{c}$. Viral load declines whenever $v_{\alpha}^{-}(0), v_{\alpha}^{+}(0)>$ $\frac{P}{c}$ as depicted in Fig. 2. This represents a constraint on the initial condition to the solution to be consistent with the expected immune recovery expected in individuals under ART. 


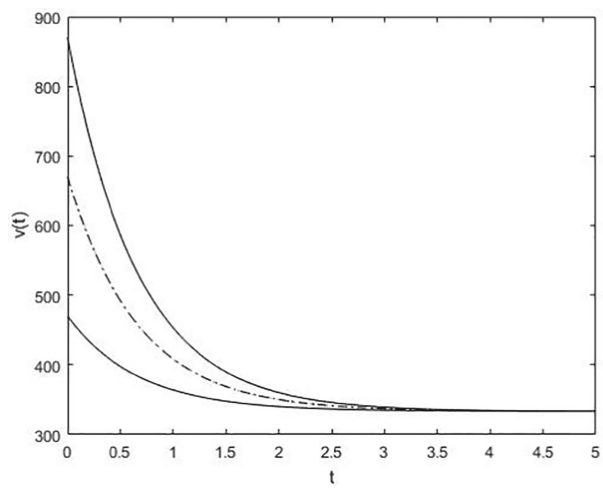

Fig. 2. Viral load as a contractive fuzzy process for $P=500$ virions/day, $c=1.5$ /day and $V_{0}=(470 ; 670 ; 870)$ virions $[18,19]$.

\subsection{Fuzzy Interactive Differential Equation via Fréchet Derivative}

Natural history of HIV suggests that after the primary infection, a large phase is observed where the viral concentration remains almost constant $[7,20]$. This set-point level can be represented in (19) as $\frac{d V}{d t}=0$, that is, $P=c V_{0}$ when treatment is started. This means the viral production rate $P$ may be considered linearly correlated to initial viral concentration $V_{0}$.

We will assume that there is $A \in \mathbb{R}_{\mathcal{F}}$ such that $V(t)=\Psi_{A}(p(t), q(t))=$ $p(t) A+q(t)$ for all $t \geq 0$. We will also assume that $p, q: \mathbb{R} \rightarrow \mathbb{R}$ are differentiable. Then, the FIVP corresponding to (19) is given by

$$
\left\{\begin{array}{l}
V^{\prime}(t)=P-\Psi_{A} c V \\
V(0)=V_{0} \in \mathbb{R}_{\mathcal{F}(A)}
\end{array}\right.
$$

where $P \in \mathbb{R}_{\mathcal{F}(A)}$, that is, there are $p_{1}, p_{2} \in \mathbb{R}$ such that $P=p_{1} A+p_{2}=$ $\Psi_{A}\left(p_{1}, p_{2}\right)$, and $c \in \mathbb{R}$ is constant. Note that, in this case, we have that

$$
P=\frac{p_{1}}{p_{0}}\left(p_{0} A+q_{0}\right)+\left(p_{2}-\frac{p_{1} q_{0}}{p_{0}}\right)=\frac{p_{1}}{p_{0}} V_{0}+\left(p_{2}-\frac{p_{1} q_{0}}{p_{0}}\right)
$$

that is, $P$ is linearly correlated to $V_{0}$. Theorem 4 ensures that

$$
V^{\prime}(t)=\Psi_{A}\left(p^{\prime}(t), q^{\prime}(t)\right)=p^{\prime}(t) A+q^{\prime}(t) .
$$

Then, (24) can be rewritten as

$$
\Psi_{A}\left(p^{\prime}(t), q^{\prime}(t)\right)=\Psi_{A}\left(p_{1}, p_{2}\right)+c \cdot{ }_{A}(p(t), q(t))
$$

and by the linearity of $\Psi_{A}$, we have that

$$
\Psi_{A}\left(p^{\prime}(t), q^{\prime}(t)\right)=\Psi_{A}\left(p_{1}-c p(t), p_{2}-c q(t)\right) .
$$


As $A \in \mathbb{R}_{\mathcal{F}}$ is non-symmetric, the operator $\Psi_{A}$ is injective, so that (28) is equivalent to two real systems, given by

$$
\left\{\begin{array}{l}
p^{\prime}(t)=p_{1}-c p(t) \\
p(0)=p_{0}
\end{array}\right.
$$

and

$$
\left\{\begin{array}{l}
q^{\prime}(t)=p_{2}-c q(t) \\
q(0)=q_{0},
\end{array}\right.
$$

whose solutions are given by $p(t)=\left(p_{0}-\frac{p_{1}}{c}\right) e^{-c t}+\frac{p_{1}}{c}$ and $q(t)=$ $\left(q_{0}-\frac{p_{2}}{c}\right) e^{-c t}+\frac{p_{2}}{c}$, respectively. Therefore, the solution of (24) is given by

$$
V(t)=\left[\left(p_{0}-\frac{p_{1}}{c}\right) e^{-c t}+\frac{p_{1}}{c}\right] A+\left(q_{0}-\frac{p_{2}}{c}\right) e^{-c t}+\frac{p_{2}}{c}, \forall t \in \mathbb{R} .
$$

Since $V(t)=p(t) A+q(t)$, we have that $\operatorname{diam}(V(t))=\operatorname{diam}(p(t) A) \rightarrow$ $\operatorname{diam}\left(\frac{p_{1}}{c} A\right)$ when $t \rightarrow \infty$. Moreover, if $A \in \mathbb{R}_{\mathcal{F}}$ is such that $0 \in[A]_{1}$, then we can consider that $A$ is a fuzzy number around 0 . In this case, we may expect that viral load to be around $\frac{p_{2}}{c}$ when $t \rightarrow \infty$. Once $P=p_{1} A+p_{2}$, this result coincides with the classic case when $P \in \mathbb{R}$.

Lastly, we have three cases to consider:

i) $c<\frac{p_{1}}{p_{0}}$ :

In this case, $p^{\prime}(t)=-c\left(p_{0}-\frac{p_{1}}{c}\right) e^{-c t}>0$, that is, $\operatorname{diam}(V(t))=$ $\operatorname{diam}(p(t) A)$ is an increasing function, as depicted in Fig. 3. Therefore, the fuzziness of $V$ increases with time, as seen in the expansive case described in Subsection B.

ii) $c=\frac{p_{1}}{p_{0}}$ :

In this case, $p^{\prime}(t)=0$, that is, $\operatorname{diam}(V(t))=\operatorname{diam}(p(t) A)=\operatorname{diam}\left(\frac{p_{1}}{c} A\right)$ is constant with time. Therefore, the fuzziness of $V$ remains constant, as depicted in Fig. 4.
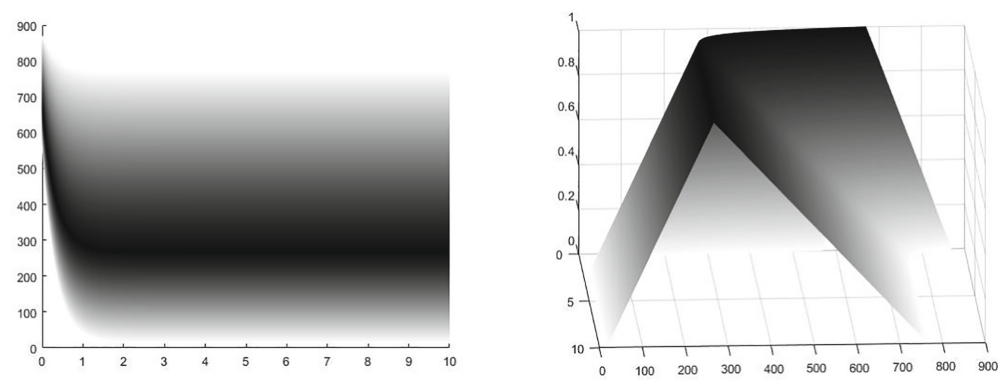

Fig. 3. Viral load for $A=(-0.5 ; 0 ; 1), V_{0}=(470 ; 670 ; 870), c=3 /$ day, $p_{0}=1500$, $q_{0}=670, p_{1}=1500$ and $p_{2}=800[18,19]$. 

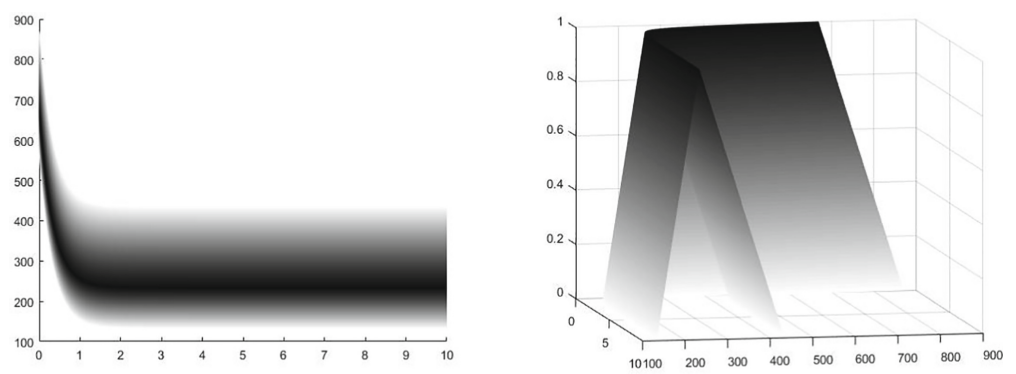

Fig. 4. Viral load for $A=(-0.5 ; 0 ; 1), V_{0}=(470 ; 670 ; 870), c=3 /$ day, $p_{0}=1500$, $q_{0}=670, p_{1}=600$ and $p_{2}=700[18,19]$.

iii) $c>\frac{p_{1}}{p_{0}}$ :

In this case, $p^{\prime}(t)=-c\left(p_{0}-\frac{p_{1}}{c}\right) e^{-c t}<0$, that is, $\operatorname{diam}(V(t))=$ $\operatorname{diam}(p(t) A)$ is a decreasing function. Therefore, the fuzziness of $V$ decreases with time, as depicted in Fig. 5.

When viral production rate $P$ is a real constant, that is, when $p_{1}=0$, the representation of $P$ in the space $\mathbb{R}_{\mathcal{F}(A)}$ is $P=\Psi_{A}\left(0, p_{2}\right)=0 A+p_{2} \in \mathbb{R}$. Theorem 5 ensures that, in this case, the Initial Value Problems (21) and (24) are equivalent if the initial conditions are the same.

However, only solutions (23) and (31) may coincide. As we observed previously, if $p_{1}=0$, then $\operatorname{diam}(V(t)) \rightarrow 0$ when $t \rightarrow \infty$, that is, the fuzziness of the solution vanishes with time, as represented in Fig. 6. For HIV dynamics predicting viral drop, the autocorrelated process described by Fréchet derivative is always contractive if $P \in \mathbb{R}$, being expansive exclusively when $P$ is a fuzzy number.
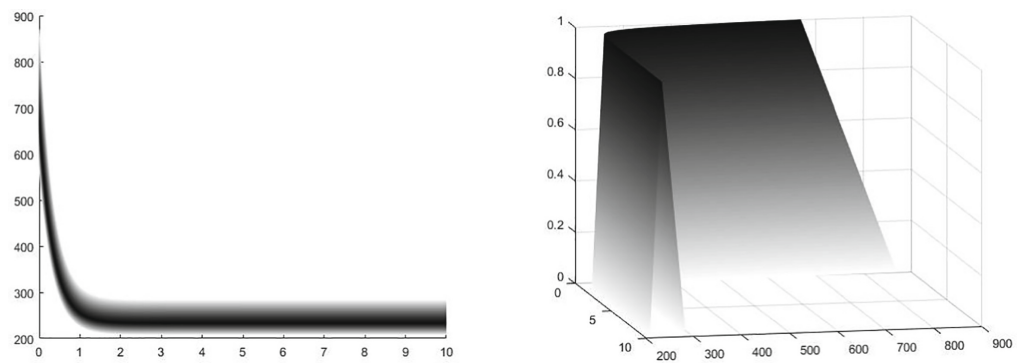

Fig. 5. Viral load for $A=(-0.5 ; 0 ; 1), V_{0}=(470 ; 670 ; 870), c=3 /$ day, $p_{0}=1500$, $q_{0}=670, p_{1}=150$ and $p_{2}=700[18,19]$. 

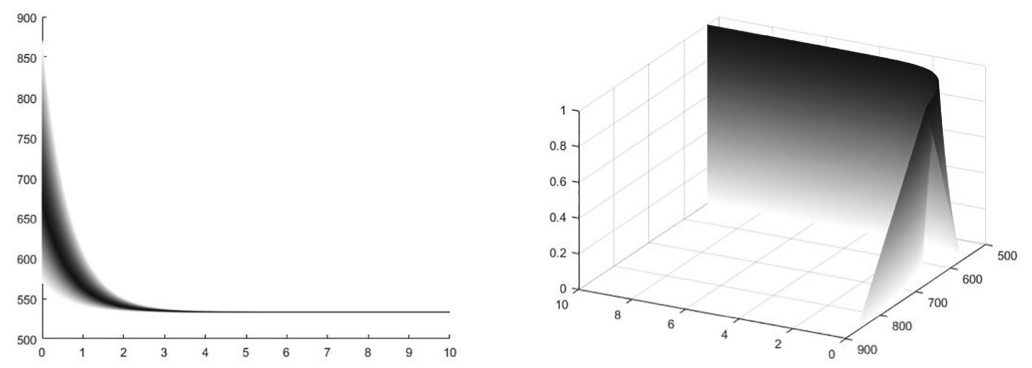

Fig. 6. Viral load for $A=(-0.5 ; 0 ; 1), V_{0}=(570 ; 670 ; 870), c=1.5 /$ day, $p_{0}=200$, $q_{0}=670, p_{1}=0$ and $p_{2}=800[18,19]$.

\section{Final Comments}

In this work we presented an HIV dynamics for individuals under ART as an application of two different approaches from fuzzy set theory: differential equation via interactive derivative and differential equation via Fréchet derivative. Biological processes may be considered as processes with memory, or from the point of view of fuzzy interactivity, autocorrelated processes. Viral dynamics was considered as an autocorrelated process in this manuscript.

Differential equation via interactive derivative provided two different types of solutions: the contractive and the expansive one. The underlying memory coefficient determines if the fuzziness of the solution decreases with time, as in the first case, or increases, as in the second case. Viral dynamics predicted a drop on viral load in bloodstream as was also observed in the two cases. For interactive derivative, viral production rate $P$ was a real constant and it was not related to the stability of the solution.

Diferential equation via Fréchet derivative provided three different types of solution: the contractive, the expansive and also a third kind, that one with constant fuzziness with time. Modelling HIV via Fréchet derivative allowed us to evaluate the viral production rate $P$ as a fuzzy number linearly correlated to $A \in \mathbb{R}_{\mathcal{F}}$. This was adopted due to the set-point that seropositive individuals remain after the primary infection, according to the natural history of HIV. It suggests that the viral production rate is linearly correlated to initial viral load $V_{0}$, with the coefficient $\frac{p_{1}}{p_{0}}$. The lower this coefficient is in relation to clearance rate $c$, the lower is the fuzziness of viral load in bloodstream. It also means that the representation of $P$ on the space $\mathbb{R}_{\mathcal{F}(A)}$ determined the fuzziness of the solution $V$.

Furthermore, for HIV dynamics presented in this work, when $P$ is a real constant, the FIVP determined by differential equation with Fréchet derivative is equivalent to the FIVP determined by differential equation with interactive derivative. In this case, only the solutions (23) and (31) coincide and $\operatorname{diam}(V(t)) \rightarrow 0$ when $t \rightarrow \infty$. We can conclude that, for HIV dynamics, the interactive process determined by Fréchet derivative has an underlying memory coefficient in $(0,1)$, that is, only provides solution whose fuzziness vanishes with time. 
Acknowledgements. This research was partially supported by $\mathrm{CNPq}$ under grant no. 306546/2017-5, and 142309/2019-2 and FAPESP under grant 2016/26040-7.

\section{References}

1. Arafa, A., Rida, S., Khalil, M.: Fractional modeling dynamics of HIV and CD4+ T-cells during primary infection. Nonlinear Biomed. Phys. 6(1), 1 (2012)

2. Arafa, A., Rida, S., Khalil, M.: A fractional-order model of HIV infection with drug therapy effect. J. Egypt. Math. Soc. 22(3), 538-543 (2014)

3. Barros, L.C.d., Bassanezi, R.C., Lodwick, W.A.: A first course in fuzzy logic, fuzzy dynamical systems, and biomathematics: theory and applications (2017)

4. Bede, B., Gal, S.G.: Generalizations of the differentiability of fuzzy-number-valued functions with applications to fuzzy differential equations. Fuzzy Sets Syst. 151(3), 581-599 (2005)

5. Bede, B., Stefanini, L., et al.: Generalized differentiability of fuzzy-valued functions. Fuzzy Sets Syst. 230(1), 119-141 (2013)

6. Carlsson, C., Fullér, R., et al.: Additions of completely correlated fuzzy numbers. In: 2004 IEEE International Conference on Fuzzy Systems (IEEE Cat. No. 04CH37542), vol. 1, pp. 535-539. IEEE (2004)

7. Coutinho, F.A.B., Lopez, L., Burattini, M.N., Massad, E.: Modelling the natural history of HIV infection in individuals and its epidemiological implications. Bull. Math. Biol. 63(6), 1041-1062 (2001)

8. De Barros, L.C., Santo Pedro, F.: Fuzzy differential equations with interactive derivative. Fuzzy Sets Syst. 309, 64-80 (2017)

9. Dubois, D., Prade, H.: Additions of interactive fuzzy numbers. IEEE Trans. Autom. Control. 26(4), 926-936 (1981)

10. Esmi, E., Santo Pedro, F., de Barros, L.C., Lodwick, W.: Fréchet derivative for linearly correlated fuzzy function. Inf. Sci. 435, 150-160 (2018)

11. Herz, A., Bonhoeffer, S., Anderson, R.M., May, R.M., Nowak, M.A.: Viral dynamics in vivo: limitations on estimates of intracellular delay and virus decay. Proc. Natl. Acad. Sci. 93, 7247-7251 (1996)

12. Jafelice, R.M., Barros, L., Bassanezi, R.: Study of the dynamics of HIV under treatment considering fuzzy delay. Comput. Appl. Math. 33(1), 45-61 (2014)

13. Jafelice, R.M., Silva, C.A., Barros, L.C., Bassanezi, R.C.: A fuzzy delay approach for HIV dynamics using a cellular automaton. J. Appl. Math. 2015 (2015)

14. Jafelice, R.M., Barros, L.C., Bassanezi, R.C.: A fuzzy delay differential equation model for HIV dynamics. In: IFSA/EUSFLAT Conference, pp. 265-270 (2009)

15. Laiate, B., Jafelice, R.M., Esmi, E., Barros, L.C.: An interpretation of the fuzzy measure associated with choquet calculus for a HIV transference model. In: Kearfott, R.B., Batyrshin, I., Reformat, M., Ceberio, M., Kreinovich, V. (eds.) IFSA/NAFIPS 2019. AISC, vol. 1000, pp. 306-317. Springer, Cham (2019). https://doi.org/10.1007/978-3-030-21920-8_28

16. Nelson, P.W., Mittler, J.E., Perelson, A.S.: Effect of drug efficacy and the eclipse phase of the viral life cycle on estimates of HIV viral dynamic parameters. J. Acquir. Immune Defic. Syndr. (1999) 26(5), 405-412 (2001)

17. Notermans, D.W., Goudsmit, J., Danner, S.A., De Wolf, F., Perelson, A.S., Mittler, J.: Rate of HIV-1 decline following antiretroviral therapy is related to viral load at baseline and drug regimen. Aids 12(12), 1483-1490 (1998)

18. Perelson, A.S., et al.: Decay characteristics of HIV-1-infected compartments during combination therapy. Nature 387(6629), 188 (1997) 
19. Perelson, A.S., Nelson, P.W.: Mathematical analysis of HIV-1 dynamics in vivo. SIAM Rev. 41(1), 3-44 (1999)

20. Perelson, A.S., Neumann, A.U., Markowitz, M., Leonard, J.M., Ho, D.D.: HIV-1 dynamics in vivo: virion clearance rate, infected cell life-span, and viral generation time. Science 271(5255), 1582-1586 (1996)

21. Puri, M.L., Ralescu, D.A.: Differentials of fuzzy functions. J. Math. Anal. Appl. 91(2), 552-558 (1983)

22. Santo Pedro, F., Esmi, E., de Barros, L.C.: Calculus for linearly correlated fuzzy function using Fréchet derivative and Riemann integral. Inf. Sci. 512, 219-237 (2020)

23. Simões, F.S.P., et al.: Sobre equações diferenciais para processos fuzzy linearmente correlacionados: aplicações em dinâmica de população (2017)

24. Stefanini, L., Bede, B.: Generalized hukuhara differentiability of interval-valued functions and interval differential equations. Nonlinear Anal. Theory Methods Appl. 71(3-4), 1311-1328 (2009)

25. Wasques, V., Laiate, B., Santo Pedro, F., Esmi, E., Barros, L.C.: Interactive fuzzy fractional differential equation: application on HIV dynamics. In: Proceedings on Uncertainty in Knowledge Bases: 18th International Conference on Information Processing and Management of Uncertainty in Knowledge-Based Systems, Lisboa, Portugal, 15-19 June (2020)

26. Zadeh, L.A.: Fuzzy sets as a basis for a theory of possibility. Fuzzy Sets Syst. 1(1), 3-28 (1978) 